Progress in the management of solid tumours

Series editor: $M r$ Colin D Fohnson, Department of Surgery, Southampton General Hospital, Southampton SO9 4WX, UK

\title{
Cell and molecular mechanisms of pathogenesis and treatment of cancer
}

\author{
David A Rew
}

\begin{abstract}
Summary
Surgery remains the mainstay of treatment for most classes of human solid tumours, with the principal exception of lymphomas, but it is insufficient in many cases to guarantee cure. With few exceptions, recurrent and metastatic solid tumours continue to defy attempts to develop effective adjuvant therapies. Recent insights into tumour biology reveal an increasingly complex picture of cell and molecular processes which confer heterogeneity and resistance to treatment upon tumours. These insights may also yield new targets for more effective adjuvant therapies.
\end{abstract}

Keywords: cell biology; pathogenesis; drug resistance; cancer
Our understanding of the biology of solid tumours has made dramatic progress in recent years. This knowledge has yet to translate into more effective nonsurgical therapies. In this review, I have distilled from the grand scope of current research some of the key themes in cancer biology which help us to understand the complexity of the therapeutic problem and the reasons for treatment failure in advanced and recurrent disease. Our expectations of the magic bullet around the corner need to be tempered by a realistic appraisal of the subtlety and sophistication of the biological problem. I will resist the common temptation to claim that every new insight or measurement of a biomarker heralds a 'cancer breakthrough'. Treatment gains are likely to be incremental rather than dramatic as cell and molecular biology advances.

\section{Modern concepts in cancer biology}

\section{TUMOUR COMPLEXITY}

Most tumours display considerable complexity of structure and function. Heterogeneity is the display of diversity. It is a fundamental feature of biological systems and is a major impediment to the understanding of the behaviour of solid neoplasms at the tissue, cellular, genetic, tumour and organ levels and the response of tumours to adjuvant treatment. ${ }^{1}$

The observer can readily appreciate spatial and structural heterogeneity in tumours at the microscopic level in tissue sections, in the proportions of tumour cells, stromal cells and supportive elements of various lineages. The three-dimensional architecture of tumours is also complex. This may manifest as a proliferative face or edge, with hypoxic or necrotic areas elsewhere.

This structural complexity has major implications for clinicopathological research, rendering many studies in which single measurements are made of tumour markers of dubious value. Indeed, intratumour variation for selected biomarkers may exceed the intertumour variation, thus invalidating some population-based prognostic studies of tumour markers.

CHARACTERISTICS OF MALIGNANT CHANGE

Solid tumours are frequently characterised by biological aggressiveness. This aggressiveness manifests as deregulated growth, local invasion, metastasis, local damage (eg, obstruction) and systemic effects (eg, anorexia, weight loss).

A single abnormal cell can grow into a lethal tumour. It is now clear that the malignant mechanism is not a single switch or event converting the normal to the malignant state, but rather a staged progression of genetic and functional changes in the cell which may be accelerated by environmental interactions and hereditary genetic changes. The existence and importance of cumulative genetic damage in the development of malignancy has been particularly well studied in colorectal cancer, with a cascade of genetic changes between normal mucosa, adenomas and invasive cancer. Hereditary genetic defects seem able to bypass some of the stages in carcinogenesis, thus accelerating the stages of molecular degeneration and promoting the early development of malignancy, as in familial polyposis coli. ${ }^{2}$ Even without extrinsic interference, genetic damage and mutation appear to accumulate with repeated cell division, particularly in the proliferative epithelia which frequently develop malignancy.

TIME AS A FACTOR IN TUMOUR BIOLOGY

All biological systems change over short and long periods of time, and the biology of tumours changes continuously both in space and time. Many biomarkers are key enzymes or functional proteins such as growth factor receptors, oncoproteins, which act dynamically and which can turn over rapidly (seconds,
Department of Surgery, University of Road, Leicester LE3 9QP, UK

D A Rew

Accepted 9 July 1997 


\begin{tabular}{|l|}
\hline $\begin{array}{l}\text { Changes in cell processes in } \\
\text { neoplasia }\end{array}$ \\
\hline - deregulated cell proliferation \\
- deregulated apoptosis \\
- deregulated gene and protein \\
expression \\
- disordered signalling \\
- altered cell adhesion \\
\hline
\end{tabular}

Box 1

\begin{tabular}{|l|}
\hline $\begin{array}{l}\text { Behavioural characteristics of } \\
\text { malignant tumours }\end{array}$ \\
\hline - local growth (proliferation > cell loss) \\
- local regression (cell loss $>$ \\
proliferation) \\
- local invasion \\
- metastasis \\
- abnormal secretion (growth factors, \\
signals, hormones) \\
- 'mass' effects (weight loss, anorexia) \\
\hline
\end{tabular}

Box 2

\begin{tabular}{|l|}
\hline Time as a variable in tumour \\
biology
\end{tabular}

Box 3 minutes or hours) within the cell. Over longer time periods, (days to weeks), the physical and functional characteristics of a tumour change significantly.

The passage of time may be measured in cell and tissue terms by cell replication and the passage of cell generations. The incidence of most forms of cancer increases progressively with age. This observation suggests that cumulative genetic damage is the key to the malignant genotype.

Why do we acquire cancer? The development of some forms of cancer is certainly promoted by cumulative environmental hazards which may be overt (such as lung cancer from cigarette tars) or covert (such as natural background radiation). These factors contribute to cumulative degenerative damage to the genome with the passage of time. We regard neoplastic, cancerous change as the acquisition of new and damaging properties by cells. However, the emergence of malignancy through cumulative genetic damage may be an unavoidable intrinsic process, rather than a disease superimposed from outside upon innocent tissues. Given the huge numbers of cells produced by proliferative tissues, dysfunctional cells are likely to be generated frequently throughout life. Most do not survive.

The aetiology of many tumours is degenerative at the cellular and genetic levels. The emergence of features of the malignant phenotype (invasion, metastasis) may be a reversion to a less highly regulated state of cell function and control. It may be that only the most immunologically evasive, and subtly abnormal cells will survive to exhibit the features of uncontrolled proliferation, deregulated cell death, invasion and metastasis. Malignancy may then be the statistical and biological price to be paid for massive and rapid cell and tissue turnover throughout life.

\section{Cancer and evolution}

We are now living longer than nature seems to have intended. Evolutionary and reproductive pressures and natural selection refined over aeons have determined the emergence of the complex biological processes within cell, tissue and organ and have imposed higher degrees of order and specialist function upon them to ensure life, reproduction and species survival. The immunological system has emerged as an extraordinarily sophisticated mechanism to cope with infection. We do not appear to have evolved a specific system to deal with malignancy.

The concept of degenerative genomic damage may explain both the absence of a dramatic immunological response to most tumours, and the observation that nature has not eliminated cancer as a harmful biological process through the evolutionary power of natural selection. Most tumours arise in the postreproductive years, at which point, in population terms, there has not been evolutionary or genetic survival pressure to develop resistance mechanisms to malignancy.

\section{Telomere loss and telomerase function}

Cell replication confers age upon the cell during repeated mitosis, through the loss of repetitive DNA from the telomeres at the ends of chromosomes each time a cell divides. Chromosomes shorten with each replication in normal cells with the loss of short repeat sequences of DNA primer fragments from the telomeric complexes at each end of the chromosomes. This mechanism imposes a limit on the number of divisions that a cell can make before the replicative mechanism is terminally damaged. Telomere shortening appears to confer a biological clock, a proliferative lifespan, and senescence, upon normal cells.

Telomerase may play a key role in the immortalisation of cancer cells. It does not appear to be expressed in normal human somatic cells. It restores the length of telomeres and transforms stem line and neoplastic cells such that they acquire an extended proliferative lifespan, as in testicular germ line cells. ${ }^{3}$ The recent discovery of this mechanism and the role of telomerase, provides one example of how simple, intrinsic, repetitive damage consequent upon normal processes can have profound consequences for the replicating cell.

TUMOUR GROWTH, CELL PROLIFERATION AND THE CELL CYCLE

Tumour growth is the product of an imbalance between cell production and cell loss, which varies considerably with time and changing environmental factors. Tumour cell populations are a complex mixture of proliferating, quiescent, noncycling and apoptotic cells. Cell proliferation alone is thus insufficient to describe tumour growth.

One consequence of this balancing act is that rapid cell proliferation does not alone determine rapid tumour growth. For example, a small, rapidly growing primary or metastatic tumour may have a normal cell proliferation rate but minimal cell loss. Conversely, a large tumour with a high cell production rate may have static volume growth because of its equally high rate of cell loss. 


\begin{tabular}{|l|}
\hline $\begin{array}{l}\text { Types of heterogeneity within } \\
\text { tumours }\end{array}$ \\
\hline - cell composition and function \\
(stromal, tumour, vascular, \\
inflammatory) \\
- tumour cell composition (DNA \\
content, stem line, degrees of \\
differentiation) \\
- $2 \mathrm{D}$ and 3D micro-architecture \\
temporal (variable change with time) \\
- physiological (hypoxia, acidity, \\
interstitial pressures) \\
- genetic structure and function, gene \\
expression (eg, multidrug resistance) \\
- cell cycle asynchrony \\
- quiescence, proliferation and apoptosis \\
intertumour variation; between \\
tumours of a class or a tisssue of origin
\end{tabular}

Box 4
The cell cycle

The processes in cell proliferation and tumour cell population dynamics are clarified by the well established cell cycle concept. Most somatic cells reside in the G0/G1 resting state, with a normal diploid DNA content, whence they may become quiescent or terminally differentiated. The diploid 46 chromosome set is duplicated during the $S$ (synthetic) phase, which lasts in the order of 15 hours. They enter a short, unstable tetraploid G2 state for a few hours prior to mitosis. The expression of many proteins vary between quiescent and proliferating cells, and fluctuate through the cell cycle with DNA synthesis and cell division.

The proportion of cells in the $S$ phase at any time can be measured by laser based flow or scanning cytometry, and is a crude measure of the proliferative activity of the sample. The growth fraction is the proportion of cells capable of proliferation in the total cell population.

\section{Cell cycle control and proliferation}

The regulation of the cell cycle is complex but precise and highly conserved by evolution. Considerable advances have been made in the measurement and understanding of the molecular processes underlying normal and tumour cell cycle control. ${ }^{45}$ Cell cycle regulation involves complex and dynamic structural relationships between proteins such as p21, p53, cyclins, cyclin-dependent protein kinase (cdk) proteins and proliferating cell nuclear antigen, ${ }^{4}$ which impose a logical and temporal discipline on the cell cycle. The fidelity of proliferation is so important to the survival of the normal organism that complex homeostatic controls and fail-safe mechanisms are likely to have evolved to achieve this end.

Members of the cyclin (A-H) and cdk families of proteins go through a complex sequence of synthesis, association and degradation as the cell cycle progresses. For example, cyclin $\mathrm{C}$ is closely associated with spindle microtubules during mitosis, while cyclin $\mathrm{B}$ resides in the Golgi apparatus. Other proteins, including p15, p16, p18, p19, p21, p27 and ubiquitin, function in the inhibition, degradation and proteolysis of these regulatory switches. The cyclin system ensures secure and efficient mitotic reproduction of normal cells. Signal transduction around the cell is an important feature of cell cycle regulation, and the various cyclins also localise in different sites in the cell. Deregulation of cell cycle control appears frequently to be mediated through loss of the checkpoint to proliferation in G1 associated with one or other proteins in the cyclin $\mathrm{D} / \mathrm{Cdk} 4 / \mathrm{p} 16 / \mathrm{Rb}$ multiprotein complex.

The immense subtlety and complexity of cell regulation provides particular challenges to the development of effective anticancer drugs. Disruption of one pathway is likely to lead to complex re-adjustments and 'workarounds' in other pathways, endowing considerable resilience upon the cell, and, paradoxically, possibly endowing new characteristics of resilience, resistance and virulence upon surviving cells during therapy.

\section{Cell division}

Cell division is important as a target for disruption by drugs such as the mitotic spindle poisons (the vinca alkaloids). Specific check-points exist at various points in the cell cycle and also ensure that all criteria for normal cell division, such as the precise alignment of chromosomes during mitotic metaphase, are met before cell division can proceed. Order is imposed on the distributing chromosomes by the formation of a bipolar spindle. Each set of chromosomes becomes attached to one pole of the spindle by filamentous, contractile protein microtubules. Microtubule elongation from tubulin subunits is initiated at a centrosome at each end of the spindle. Within each condensed chromosome are regions of microtubule attachment known as centromeres, and composed of repetitive DNA base sequences. The attachment is mediated through kinetochores. Chromosome pairs become evenly aligned on the equator of the spindle prior to microtubule contraction and chromosome separation. The taxols appear specifically to disrupt microtubule-mediated processes.

Cell division is also important as a vehicle for genetic disruption during normal and malignant cell division, and in the genesis of aneuploidy.

\section{ANEUPLOIDY}

Allelic (chromosomal fragment) loss and gain

The aging of the genetic and molecular machinery of the cell may manifest as aneuploidy in cancerous cells. ${ }^{6}$ The absolute or functional loss of alleles is a commonly reported genetic correlate of malignant change. Up to $75 \%$ of solid tumours display aneuploidy, which is abnormal genomic content characterised by the acquisition (or occasional loss) of multiples of chromosomes or chromosome fragments. 
Diploidy is the possession of the normal, species-specific complement of two pairs of haploid ( $n=23$ in man) chromosome sets. Where chromosome fragment (allelic) loss occurs at one site, it may be balanced either by translocations or DNA gains elsewhere in the chromosomal make-up. Hypoploidy results from allelic and regulatory gene loss and imbalance. Aneuploidy can also arise from the gain of complete chromosomal sets. Exact multiples of the haploid number are described by specific terms, for example, the triploid (69 chromosomes) or tetraploid (92 chromosomes) states. The measurable ratio of abnormal to normal cellular DNA content is the DNA index of the cell, tissue or tumour. DNA indices of up to 3.0 (hexaploidy) have been reported.

\section{Molecular mechanisms causing aneuploidy}

Chromosomal disorder and aneuploidy are most likely to arise during mitosis or meiosis, when chromosomes are being shuffled and replicated. There are a number of different points during both processes at which chromosomal aneuploidy may arise. These errors may be induced by drugs acting as mitotic poisons including viruses, cytotoxic agents such as dimethylhydrazine, vincristine, diethylstilboestrol, and colchicine, and ionising radiation.

Germ-line abnormalities will give rise to constitutional aneuploidy throughout the body. Meiosis provides a number of points at which aneuploidy may arise Uneven chromosome alignment, abnormal kinetochore development and function, failure of pair separation (nondysjunction), or errors of spindle orientation and contraction may lead to uneven DNA distribution and hence to aneuploidy during both mitosis and meiosis. Inaccurate alignment at synaptosomal complexes results in the production of unequal chromosome lengths once crossover has occurred. Nonhomologous chromosomes may pair up, again producing uneven exchange of DNA.

\section{CELL LOSS}

Cell loss is a key process in tumour biology. From the perspective of the tumour cell, it may be inadvertent, through hypoxia, immunological response, therapeutic action, exfoliation or shedding, or deliberate. The discrepancy between cell production rates and tumour growth rates suggest that up to $95 \%$ of tumour cells may be lost at certain phases in tumour growth. If this proportion is only slightly increased, to just over $100 \%$, the tumour will start to regress.

\section{Programmed cell death: apoptosis}

Apoptosis, or programmed cell death, is a normal, energy-dependent process which can be seen during embryonic development, tissue maturation, and the regulation of haematopoiesis. It proceeds so rapidly in tissues that it went unrecognised until the 1970 s. It is now recognised as a prime cause of cell loss. ${ }^{78}$

Apoptosis varies in form and proportion from tissue to tissue. The key events are cell shrinkage and rounding off, loss of adhesion, nuclear condensation and systematic chromosomal fragmentation. Crucially, the cell and nuclear fragments are rapidly phagocytosed either by adjacent cells or by macrophages. Because apoptosis is complete within a few hours, it can have dramatic consequences for the rate of tumour growth or regression, while leaving few discernible fingerprints in tissue sections. It is difficult to identify apoptosis in histological material. The recent development of direct histochemical markers of DNA damage and disruption, particularly based on the binding of the nucleotide dUTP to DNA strand breaks, apoptosing cells may now be directly identified in tissue sections.

Many molecular processes and signals appear to converge in mediating apoptosis, which consists of two sequential events. Firstly, cells are primed (the gun is loaded), after which the cell can undergo apoptosis in response to other signals (the trigger is pulled). The genes myc, ras, rb, p53, bcl-2, and bax, all appear to have important priming functions in response to intrinsic and extrinsic cell signals.

A number of molecular events precede apoptosis. These include the switching on and off of key regulatory genes and the synthesis of key proteins and enzymes. Genes implicated in the regulation of apoptosis include p53, cmyc, bcl-2, max, and bax. The interactions between these genes, the many other genes now implicated in apoptosis, and the immediate environment of the cell are undoubtedly complex. A number of genes, including bcl-2, protect against apoptosis; bcl-2 protein is found on internal cell membranes, including endoplasmic reticulum and mitochondria.

The cytokines are proteins that regulate cell differentiation through target cell receptor binding. Two particular cytokines, Fas ligand and tumour necrosis factor induce apoptosis rapidly by binding to cells. Thus, apoptosis can be controlled by factors extrinsic to the cell, and is not strictly a 'cell suicide' programme. ${ }^{8}$ 


\section{Genetic changes in tumours}

- point mutations in genes

- sequential and cumulative damage

- gene and allelic loss and gain

- chromosome disorganisation and reorganisation, translocation

- disorders of mitosis and meiosis, chromosome segregation

- gross disorders of chromosome content (aneuploidy)
Another important family of regulatory proteins in apoptosis are the interleukin-converting-enzyme-like proteases, which mediate chromosomal degradation after Fas ligand or tumour necrosis factor binding to the cell membrane. This mechanism is blocked by the protein product of the gene bcl- 2 .

Malfunction of this complex and delicately balanced regulatory system is likely to lead to abnormalities of tissue growth, and is described by Nagata as a double-edged sword. ${ }^{8}$ For example, it is possible that tumour necrosis factor produced by tumours leads to cell loss in normal tissues (and to cachexia).

\section{GENE MUTATIONS AND GENETIC DISORDER}

The genetic disturbances in malignant cells may be classified into three groups. Primary abnormalities of the genome, such as point mutations in regulatory genes and oncogenes, may produce critical neoplastic changes. Secondary abnormalities, such as gene amplification and chromosome duplication, may arise within an unstable cell genome. The reduplication of one or more whole chromosomes or fragments may occur. Amplification of one or more individual genes may lead to the production of large numbers of copies. Amplification of DNA sequences of unknown function ('junk') may occur within chromosomes, but with no change in the numbers of normal genes. Abnormalities may also be detectable in cells in which viruses or bacteriophages have become incorporated and or multiplied in continuity with host chromosomes. Cytogenetic noise is another state of DNA abnormality. It relates to the many chromosomally unstable tumour cells which are temporarily detectable during tumour growth but have no survival advantage.

Genetic heterogeneity is the foundation of Darwinian evolutionary competition, of species diversity, and of malignant change. Patients with cancer display genetic differences between the parent genotype and the tumour, and those differences vary with time, with growth and with site within the tumour. Moreover, there is no one point at which a tumour can be considered to have achieved its definitive genotype. Genetic instability may lead to many different cell lines, or clones, which may have better or worse survival characteristics.

Structural genetic disorder down to the level of single base-pair mutations leads to regulatory protein dysfunction and to malignancy. Cumulative point mutations in DNA lead to increasing genetic instability. At the chromosomal level, genetic disorder may arise through a number of different mechanisms. These include gene loss, gene amplification, and DNA methylation. This disorder gives rise to pluripotentiality of gene function and expression.

Many specified genes and their protein products have been implicated in the sequence of events leading from normal cell behaviour to malignancy. Single genes display considerable mutational heterogeneity within populations, but many of the mutations may be functionally neutral.

The p53 system as an example

The $\mathrm{p} 53$ protein system has been well researched, ${ }^{9}{ }^{10}$ and provides many insights into molecular structure and function in normal and malignant cell regulation. In general terms, normal p53 appears to prevent progression through the cell cycle of genetically damaged cells, while mutant p53 allows uncontrolled cell cycle progression and impairs apoptosis. It is a transcription factor which is known to modulate the expression of at least seven key genes. p53 is normally present at low levels in cells, but is rapidly synthesised in response to DNA damage or hypoxia. It may then act as a common pathway or 'gatekeeper' in the coordination of damage repair, cell cycle arrest in G1, and apoptosis. Normal p53 may also mediate the effects of chemotherapy and radiotherapy.

A large number $(>2600)$ of sequence variations and point mutations in the protein of the $\mathrm{p} 53$ gene have been detected..$^{11}$ Many amino acid sequences within the $\mathrm{p} 53$ protein are known to have specific molecular functions, such as protein and DNA binding. Many key mutations of p53 in cancer cells affect p53 binding to DNA. Seemingly minor 'degenerative' changes in the genome may thus have profound consequences for cancer development.

The BRCA1 and BRCA2 genes

This is also true of the role of the tumour suppressor genes BRCA1 and BRCA2 in hereditary and sporadic breast cancer. Both produce large nucleus-associated proteins. Both copies of such genes must be inactivated (the 'two-hit' hypothesis) for cancer to develop. Familial studies, such as on $\mathrm{Li}$ Fraumeni syndrome, have elucidated the BReast CAncer (BRCA) 1 gene on chromosome 17q21, for which more than 100 mutations are now recognised. BRCA1 mutations often produce an incomplete protein product, thus facilitating BRCAl detection through the protein truncation test, which can be performed on cytoplasmic DNA amplified 
from leucocytes. Ashkenazi Jewish families are particularly at risk of transmission of one mutant of the BRCA1 gene with AG base deletion at position 185 .

Gene mutation affects tumour morphology and behaviour. The BRCA2 gene is found in sporadic breast cancer. It encodes the protein E-cadherin, which is commonly found to be mutated in lobular carcinoma. E-Cadherin functions in the junctional complexes of epithelial cells, and its dysfunction may find physical expression in the specific histomorphology of lobular carcinomas.

OTHER NUCLEOPROTEINS, ONCOPROTEINS, AND REGULATORY GENES

Many genes and cell regulatory processes are highly conserved through nature and evolution. A large array of tumour-associated genes and their protein products have now been recognised. Their disruption through mutation or allelic loss leads to disorder and malignant disfunction in key processes, including proliferation, apoptosis and signalling functions. It is not possible to review these in detail here, but many excellent reviews exist. ${ }^{11}{ }^{12}$

These regulatory genes are broadly classified as having tumour promoter and tumour suppressor functions. Many have interrelated functions. Their products include nuclear, cytoplasmic, and membrane-associated molecules. Detailed understanding of the growth factor and growth inhibitor initiated signalling pathways has revealed the extent and complexity of the interrelated roles of the oncoproteins. Malignant change may be mediated through a number of newly recognised pathways which act at various sites in the cell:

Through cell surface receptors Mutant-activated protein-tyrosine-kinase pathways may disrupt intracellular regulation, thus providing a link between extrinsic cell signals and regulatory factors (eg, vascular endothelial cell growth factor and fibroblast growth factor), cell surface receptors (eg, cadherins, catenins), cell-to-cell adhesion and oncogenesis.

Through signalling pathways to the nucleus Mutations are recognised in various intracellular signalling systems in cancer, including the TGF-beta and Ras oncoprotein systems.

Through the actin cytoskeleton Expression of the cytoskeletal proteins is commonly altered and disorganised in transformed cells. The cytoskeleton plays a key role in signalling, adhesion, binding and as a binding framework for regulatory processes.

At the nucleus The increasing number of nuclear oncoproteins include transcription factors and regulators, and chromatin regulatory proteins.

OTHER CRITICAL CELL FUNCTIONS

Many other critical cell functions contribute to the homeostasis and behaviour of the normal cell, and their subtle modulation may produce the malignant phenotype. Such processes include intra- and intercellular signalling, cell adhesion, angiogenesis, and metastatic behaviour.

\section{Cell adhesion}

A large number of cell-membrane-associated adhesion molecules have been recognised, ${ }^{1314}$ including the integrins, the cadherins, the selectins, the immunoglobulin-like proteins, and the selectin families of molecules. The transmembrane integrins bind to cytoskeletal and extracellular matrix proteins and communicate extracellular signals. The immunoglobulin-like protein family subserves a wide range of functions in immunological interactions, such as antigen recognition and complement binding. The cadherins are transmembrane proteins which mediate cell-cell interactions such as forming junctional complexes, cell orientation, and regulation of morphogenesis.

The adhesion molecules and their altered expression may be associated with a number of component processes in neoplastic behaviour, including modified growth and angiogenesis, and the capacity for detachment and metastasis. ${ }^{15}$

Cell signalling and growth factors

Cell signalling governs the imposition of structural and functional order on tissues. Cell-to-cell contact as mediated by adhesion molecules constitutes one form of signalling. Signalling is also effected both by the secretion of signal molecules, for local and distant communication. Classes of secretory molecules include growth factors, which are important signal mechanisms. Examples include epidermal, and platelet-derived factors, while hormones are examples of distance signalling molecules. One simple example is the role of the oestrogens in promoting the growth of some breast carcinomas. 


\section{Angiogenesis}

New vessel formation is an important process both for primary and metastatic tumour growth. The processes of promotion and inhibition are under careful study as attractive targets for therapeutic strategies. A number of regulatory proteins have been identified, including angiostatin, ${ }^{16}$ and several families of protein-tyrosine-kinases have been particularly associated with angiogenesis. These include vascular endothelial cell growth factor and fibroblast growth factors. Many solid tumours are known to produce vascular endothelial cell growth factor, for example, in response to hypoxia. ${ }^{12} 1718$

\section{Metastasis}

Metastasis is the process which above all determines the aggressiveness of the tumour and the clinical outcome. Metastasis encompasses a number of processes, including detachment of viable cells from the primary tumour (loss of E-cadherin function appears to be a necessary precursor of cell detachment, invasion and metastasis); survival during transmission and migration to the metastatic site; adhesion and establishment at the metastatic site; and proliferation and angiogenesis at the new site.

The treatment of established metastases provides particular therapeutic challenges. There may be significant differences between primary tumours and their metastases. The latter may not be simple clones of the parent, primary tumour. They may have different genetic and functional characteristics. They must develop in a different immunological and nutritional environment, such as a lymph node or in bone marrow.

In many cases, metastasis is not an entirely random process. Many classes of tumour can be defined in part by their relatively ordered patterns of metastasis. For example, colorectal tumours consistently progress via the loco-regional lymph nodes to the liver, while ovarian tumours spread trans-peritoneally. The presence of such order implies regulatory mechanisms which may be under specific genetic controls, such as the nm23 gene. ${ }^{15}$

\section{Cell and molecular mechanisms in treatment}

A key aspect of human solid tumour biology and behaviour is the generic lack of long-term response to existing treatment modalities. Early response and tumour shrinkage is usually followed by the re-emergence of aggressive disease.

All existing treatments cause damage to normal cells and tissues, often to a degree which prevents effective cancer treatment. The challenge for tumour biologists is to find features of tumour cells which are sufficiently distinctive from normal cells such that the therapeutic ratio is substantially widened and the side-effects of treatment are minimised.

\section{TREATMENT STRATEGIES}

The diversity of processes at play in the malignant phenotype, and the complexity of damage-resistance mechanisms, provides great scope for the development of treatment strategies, and for the re-assessment and better use of existing treatment modalities. Treatment strategies may be considered in conventional and novel categories.

Conventional drug treatments have historically been targeted at DNA replication and cell proliferation, stemming from the belief that cell production alone was the key to cancer progression. We now recognise that many of the powerful agents developed for this purpose may have secondary or alternative modes of action in interfering with other processes, and particularly in the induction of apoptosis. This opens up a new range of attractive and subtle treatment options using conventional agents in conjunction with, for example, calcium channel antagonists.

Novel treatments emerging from molecular biology research include gene transfer, viral vector and immune targeting mechanisms (see below).

THE CELL CYCLE AND THERAPEUTIC STRATEGIES

The impairment of cell proliferation has long been a key objective for adjuvant therapy. Skipper et $a l^{19}$ hypothesised that a given dose of drug kills a constant fraction of cells, regardless of the total cell mass, and that there is a broadly inverse relationship between tumour cell mass and response to therapy. Clinical response and mass reduction during therapy does not necessarily reflect a change in proliferative capacity. Residual tumour is likely to consist of the more resistant and dangerous clones which ultimately determine outcome.

Many drugs can be shown in laboratory models to act selectively in particular phases of the cell cycle, ${ }^{20}{ }^{21}$ and drugs are often defined by their mechanisms of action in relation to DNA synthesis and the cell cycle. Drug efficacy demands a 
greater drug selectivity for malignant than normal cells (the therapeutic index). Drug efficacy is a balance between damage induction and damage limitation, and relates to cell cycle controls and check points. For example, specific topoisomerase II inhibition by agents such as ICRF193 at the G2 checkpoint produces disorganised chromosome segregation in G2, while mitotic spindle poisons such as the taxol derivatives disrupt cell division.

Combinations of cell-cycle-active cytotoxic drugs have been thought to exploit kinetic differences between tissues in vivo. Unfortunately, drug strategies focussed on the disruption of proliferation have not generally been successful. There are a number of explanations for the lack of correlation between proliferation measures and clinical response. The simplicity and homogeneity of cell line and experimental animal models is often not replicated in human tumours. The outcome of therapy based upon cell cycle strategies is influenced by the complexity, asynchrony, and heterogeneity of proliferation within solid tumours. There are also unpredictable changes in patterns of proliferation during the course of the treatment, and local cell depletion acts as a potent stimulus for hitherto quiescent cells to reactivate and proliferate.

Moreover, the cell proliferation rates of normal and malignant cells are often similar. Rapid cell proliferation is a feature of many normal tissues, including haemopoietic and mucosal cells. For example, studies show that cell proliferation and cell cycle dynamics are similar in colorectal carcinomas and mucosa. ${ }^{22}$ Such studies suggest that altered proliferation rates are not a satisfactory explanation of neoplasia. Indeed, the mechanics and timing of such a complex and fundamental process as the cell cycle might be expected to be conserved between normal and abnormal cell types. Significant disorder in cell proliferation introduced by the malignant phenotype would be likely to halt the cell cycle and thus the malignant process.

APOPTOSIS AND THERAPY

Modulation of programmed cell death by adjuvant therapy may be an important determinant of clinical outcome. An increase in cell loss can be as important as a reduction in cell proliferation in determining the clinical progression of a tumour and its response to therapy.

Subtle adjustments and a small percentage change in the rate or fraction of apoptosing cells can have a profound effect on the rate of cell loss and hence of tumour growth or regression. Many of the cytotoxic drugs which disrupt the cell cycle can also induce apoptosis in tumour cells. ${ }^{24}{ }^{25}$ Drugs with a range of actions appear able to trigger apoptosis, so the cell may possess mechanisms which recognise the formation of a variety of drug-target interactions as a trigger to apoptosis. However, there remains controversy as to the significance and usefulness of apoptosis as a target for chemotherapy. Apoptosis may merely be a final common pathway for many different initial mechanisms of cell damage.

MODULATION OF TUMOUR GROWTH AS A THERAPEUTIC PROCESS

Total eradication of tumour from a live body may often be both an unnecessary and an unrealistic goal. If tumour growth can be manipulated such that the patient dies of unrelated natural causes in due course, then the therapeutic strategy can be judged to be successful.

A number of molecular mechanisms have been identified as possible targets for cell modulation. These include cell signalling pathways, enzyme systems such as the protein kinases, key oncoproteins (see below), and the mechanisms of metastasis. ${ }^{26}$ The concept of tumour modulation has implications for drug design. Modulatory agents will need to be used over considerable periods, and hence will need to be orally active and relatively free from side-effects.

An effective drug for the modulation or elimination of a solid tumour or metastasis must reach all target cells with proliferative potential, particularly tumour stem cells, despite the problems imposed by hypoxia, deficient vasculature, abnormal interstitial pressures and $\mathrm{pH}$. The drug must effectively inhibit proliferation or cell division, or induce cytostasis or apoptosis, and overcome intrinsic homeostatic and 'drug resistance' mechanisms. All this must be achieved clinically with an acceptable route, mechanism, and frequency of administration. The agent must have a satisfactory therapeutic ratio, an acceptable incidence of side-effects, and produce a demonstrable improvement in survival rather than response in controlled clinical trials. One group of agents which have demonstrated real success for such a strategy are the hormonally targeted drugs such as tamoxifen for breast cancer and luteinising-hormone-releasingfactor antagonists for prostatic cancer. 


\section{Mechanisms in chemotherapy and radiotherapy treatment failure \\ Passive mechanisms \\ - structural and genetic heterogeneity \\ - abnormal vascularity and drug access \\ - altered physiological environment (hypoxia, acidity) \\ Active mechanisms \\ - immediate: membrane-bound pumps (eg, p170-PGP); drug metabolism (eg, glutathione enzymes); DNA repair systems, topoisomerases \\ - inducible: the above systems; cell proliferation switched from quiescence; emergence of resistant cell clones}

Box 6

\section{Cell and molecular mechanisms in treatment failure}

\section{CAUSES OF DRUG TREATMENT FAILURE}

\section{Passive mechanisms of resistance}

Passive mechanisms of resistance arise from the complexity of tumour architecture and content. The anatomical heterogeneity of solid tumours creates natural resistance mechanisms to adjuvant therapy. Most solid tumours proliferate at the periphery, leaving the centre or core relatively undervascularised. As tumours enlarge, so internal blood flow and diffusion of gases and nutrients becomes less and less uniform. This prevents the even perfusion of drugs throughout the substance of the tumour. The resulting central hypoxia, acidity and raised interstitial pressure can be shown to impair drug uptake and biological activity, and hence increase resistance to therapy. ${ }^{27} 28$

Genetic heterogeneity confers resistance by producing phenotypic variations in protein and enzyme expression and function which affect the uptake and response to cytotoxic drugs or to DNA damage. Resistance to treatment is conferred de facto by the existence of a genetically diverse and pluripotential cell population. Competition for survival within the complex environment of a single tumour may be intense. As some cells are destroyed by therapy or necrosis, so the biological 'fitness' of others may manifest as changes in proliferation, drug resistance, greater capacity for DNA repair, reduced rates of apoptosis, or a greater capacity to metastasise. A natural selection process is thus at work within the growing tumour among multipotential cells, and environmental forces such as cytotoxic therapy will effect different selection pressures on different clones.

Tissue hypoxia is a particularly important consequence of structural heterogeneity for both chemotherapy and radiotherapy, and various strategies have been designed to counter it. For example, misonidazole, a drug which increases radiosensitivity in hypoxic cells, is selectively toxic for hypoxic cells in tissue culture.

\section{Active mechanisms of resistance}

Active mechanisms of resistance may be immediately available to the cell or may be inducible by noxious stimuli. All human cell lineages possess subtle, complex and reliable systems to guarantee the fidelity of reproduction, function and survival over evolutionary timescales. They possess considerable redundancy in their molecular engineering to guarantee against environmental damage. This makes cell lineages extraordinarily resilient to deliberate damage by medical intervention. Over aeons, cells have adapted to lifelong background and cosmic radiation, which in turn has prepared their resistance mechanisms for modern drugs and radiotherapy. Active mechanisms of damage resistance include:

- the expulsion of unwanted drugs from the cell (membrane-bound pumps)

- the detoxification of drugs once inside the cell (eg, the glutathione system)

- the repair of DNA damage

- the increased production, genetic upregulation and compensation for damage to specific molecules

- the switching into proliferation of previously quiescent clones to compensate for the damage and destruction of other cells

- the downregulation of apoptosis to enhance cell survival

- the selection and emergence of resistant cell clones.

Intrinsic, active, chemotherapeutic resistance mechanisms

Early success in chemotherapy is often followed by relapse and subsequent failure. Mutant, drug-resistant cell lines may be selected during the course of treatment and may supersede the drug-sensitive cells. A variety of cell functions contributes to the evolution of resistance to therapy. Intrinsic multidrug resistance mechanisms confound simple strategies for anticancer chemotherapy.

The topoisomerases

Interphase chromosomes are unravelled and the DNA is exposed for genetic activities or DNA duplication to proceed. As chromosomes condense during early mitosis, there is considerable scope for entanglement in this molecular spaghetti. The topoisomerase and DNA ligase family of protein ${ }^{29}{ }^{30}$ may be important in preventing such DNA disruption and entanglement, which might in turn predispose to aneuploidy.

The topoisomerases are nuclear, DNA-associated conformational enzymes with house-keeping functions such as the repair of strand breaks, and the facilitation of chromosome cleavage and segregation. Their inhibition by drugs such as the anthracyclines leads to cell death through the accumulation of DNA 
damage. These drugs bind to and trap human topoisomerases, levels of which increase within in a variety of cell lines as a result. This stimulation of topoisomerase expression may paradoxically confer drug resistance upon surviving cells.

\section{DNA synthesis and repair processes}

A number of DNA-associated proteins and enzymes contribute to DNA synthesis and repair in addition to the topoisomerases. DNA damage may arise spontaneously in the course of normal molecular processes, from natural or therapeutic radiation, or through chemotherapy. DNA repair imparts reproductive continuity and fidelity upon cells, and may protect against a much higher incidence of cancer in the natural world. To put this capability in context, it has been estimated that the DNA of each cell, comprising $4 \times 10^{9}$ bases, temporarily loses 10000 nucleotide bases each day from spontaneous DNA damage alone. There is a predicted efficiency of only three mistakes per 3 billion base pairs during DNA copying, in each of the $10^{14}$ cells in the human body, and in up to $10^{16}$ cell generations per human lifespan in stem cell lines (such as the haematopoietic system). Not only is the existence of such a DNA repair capability remarkable, but it highlights a profound problem in cancer therapy, because such an evolutionarily stable biological process is likely to possess considerable redundancy against DNA damage induced by clinical therapeutic manoeuvres. ${ }^{31}$

Other examples of the DNA repair system include the DNA strand mismatch repair gene $\mathrm{hMSH} 2$, defects of which are significantly associated with the emergence of colorectal cancer in the hereditary non-polyposis colorectal cancer syndrome. Protection against cell damage by ultraviolet light is afforded by the nucleotide excision repair pathway, abnormalities of which are found in xeroderma pigmentosum. Nucleotide excision repair pathway mechanisms may also repair cytotoxic drug induced damage in target cells.

\section{Cellular detoxification mechanisms}

Cellular detoxification mechanisms also contribute to drug resistance. ${ }^{32}$ Glutathione metabolism is one example of this process. Free chemical radicals such as reactive oxygen and hydroxyl groups are generated within cells as a result of radiation or drug treatment, such as with cis-platinum and doxorubicin. Glutathione is believed to play an important role in binding these moieties through oxidation-reduction reactions. This is in part mediated by enzymes such as glutathione-S-transferase and glutathione reductase. Modulation of these substances within experimental cell models can be shown to influence the resistance of cells to drug- and radiotherapy-induced damage. ${ }^{32}$

\section{Membrane-associated multidrug resistance}

The accumulation and intracellular action of cytotoxic drugs in the target cell may be modulated by a number of molecular mechanisms which confer drug resistance. These mechanisms are often referred to as the multidrug resistance phenotype. They evolved in the natural world long before the advent of chemotherapy, and it can be deduced that they are fundamental, general purpose, homeostatic mechanisms. It is thus likely that multidrug resistance will be capable of expression to varying degrees in all cells.

The multidrug resistance glycoproteins are a family of membrane-bound ATP-dependent glycoproteins of which p170 has been well characterised. ${ }^{33}$ They pump out of cells lipophilic drugs such as colchicine, adriamycin, vinca alkaloids, and daunorubicin. An increase in drug efflux may confer a degree of cytotoxic drug resistance upon the cell. Multidrug resistance gene p170 or protein expression can be found in most tumour classes in which it is sought.

The drug efflux pump may be blocked with competitive or non-competitive inhibitors. p170 is susceptible to pharmacological blockade by a variety of agents in vitro. These efflux modifiers include the anticalmodulin phenothiazines, calcium channel blockers such as verapamil, tamoxifen, quinidine, cyclosporin, $\mathrm{N}$-acetyl daunorubicin and cephalosporins. Unfortunately, the pharmacological doses of these agents required to attain clinically useful effects are unacceptable in vivo. However, new and specific multidrug resistance blocking agents are under evaluation and these may allow re-evaluation of $\mathrm{p} 170$ blockade as a practical synergistic adjunct to chemotherapy.

The p170 multidrug resistance glycoprotein pump is one of several such membrane-associated mechanisms. The multiresistance protein is one of a family of ATP-dependent multispecific organic anion transporters within epithelial and endocrine cells and macrophages. The lung resistance protein is associated with RNA in protein pores or 'vaults' on nuclear and endoplasmic reticulum membranes, and may be an important component of RNA transport between 
cell compartments and of the expulsion of unwanted substances from cells in exocytic vesicles.

\section{Tumour biology and radiotherapy}

Radiotherapy is an important component of treatment for many recurrent and advanced tumours. Ionising radiations produce damage to DNA by high energy transfer and by the production of reactive molecular species, such as superoxide radicals. Sufficiently intense irradiation will destroy all cells. The clinical effectiveness of radiotherapy depends upon achieving a maximal tumouricidal effect at sublethal doses while minimising damage to normal tissues. No regime of radiotherapy will produce completely selective killing of tumour cells while preserving normal tissues. ${ }^{34}{ }^{35}$

Radiosensitivity is a function of various factors, which include the proliferative behaviour of the tumour cells, tissue hypoxia, DNA repair mechanisms, and intracellular damage limitation processes, such as the presence of thiol compounds to mop up superoxide radicals. Cell populations respond to radiotherapy with the processes of repair, redistribution, reoxygenation and repopulation. Quiescent, noncycling cells may be less sensitive to radiation damage. Tissue hypoxia, such as is commonly found in the centre regions of solid tumours, imparts radioresistance The oxygenation of tissues may be improved during radiotherapy by hyperbaric oxygen, or by using drugs which mimic the radiosensitising effects of oxygen, such as misonidazole or bromodeoxyuridine. Cells tolerate and overcome sublethal radiation doses to varying degrees as a result of the complex interactions of many heterogenous processes, and resistant clones commonly come to be selected during treatment.

NOVEL ADJUVANT THERAPIES

Tumour targeting imposes specific design and performance characteristics upon chemotherapy. The optimum time for treatment is probably when the mass of tumour is minimal, such as at the time of putative curative surgery, when viable tumour cells and micrometastases may survive and disseminate. The number of cells is tiny in proportion to total body mass, and their distribution and location is unknown. Their certain destruction is beyond the capability of current cytotoxic drugs, because of their low specificity for tumour cells and their high incidence of side-effects for normal tissues. Therapy must therefore be extremely selective and specific for target cells, but also systemic to seek out all tumour cells. Such capabilities are characteristic of monoclonal antibodies and viruses. However, the defining antigens which might distinguish tumour from normal cells so as to allow their selective targeting have not yet been consistently identified, if indeed they exist.

\section{Immune targeting and antibody-directed therapy}

Monoclonal antibodies have considerable theoretical attractions. ${ }^{36}{ }^{37}$ Some antibodies have been produced which show sufficient specificity to supplement conventional imaging modalities in the localisation of primary tumours and metastases. Monoclonal antibodies may have a role in therapy, either alone or for the targeted delivery of a conventional cytotoxic agent or radioisotope. ${ }^{38}{ }^{39}$ However, it is not yet clear whether tumour antigenicity will be systematic or whether it will differ between tumours. Possible target antigens include tumour mucins, and oncofoetal proteins. Even if tumour cells are sufficiently antigenic for the production of specific monoclonal antibodies, there are likely to be considerable limitations to their use in practice. Problems include antigenicity, and the risk of anaphylactic and other immunological reactions. They require intravenous delivery, and the diffusion of these large molecules to the target tumour cells is likely to be imperfect. Indeed, if tumours were significantly and abnormally immunogenic, one might expect the host immunological system to deal with them much more efficiently than it already does.

\section{Gene transfer therapy}

Gene transfer therapy ${ }^{40}$ aims to introduce corrective genetic signals into abnormal cells. Gene therapy may be targeted at somatic or germ line cells. The criteria which justify research into gene therapy for any disease are that the disease process should be life-threatening but potentially reversible, that the gene should have been cloned, that a delivery system should be feasible and that there should be a measurable end point to demonstrate therapeutic gain. Candidate diseases include immune deficiency syndromes, cystic fibrosis, the haemoglobinopathies, metabolic disorders, and various forms of cancer. A variety of gene transfer techniques have been considered, including immune enhancement, the vectoring of biologicals, such as tissue-specific viruses, tissue protection 


\section{Learning points \\ - major advances have been made in understanding the genetic and molecular mechanisms underlying cancer in the past decade \\ - complexity and heterogeneity are key features of tumour biology and architecture \\ - tumour growth patterns are variable in time and place \\ - cell loss is as important as cell proliferation in tumour growth \\ - apoptosis is a key process in cell biology, and may provide new targets for therapy \\ - tumour growth modulation and the induction of stasis may be as effective strategies as those aimed at tumour elimination. \\ - the tumour cell has effective defences against radiotherapy and chemotherapy damage in DNA repair and drug resistance mechanisms \\ - similarities rather than differences between the cellular processes of tumours and normal cells handicap the development of effective therapeutic strategies \\ - new insights have yet to translate into more effective therapies}

Box 7

(eg, bone marrow), selective drug activation by specific genes, and the correction of somatic defects.

As a practical methodology for the treatment of solid tumours, it is still experimental. Firstly, the specific genes and molecular mechanisms must be identified which will achieve the desired cytotoxic or cytostatic effect. Then, for corrective therapy to be effective, the therapeutic agent must be vectored into each tumour cell, or at least every cancer stem cell, if indeed these exist and can be identified. This again poses problems of access for these large DNA moieties to the target cells.

One possible solution to the problem of tumour targeting is the use of viral vectors. Alternatively, gene therapy may allow manipulation of the immunological system to enhance its activity against tumour cells. Again, these strategies presuppose the existence of specific tumour antigenicity, eg, through cell surface receptors or the HLA phenotype.

Genetic marking may allow the detection of minimal residual disease, the characterisation of a source of recurrence, and the monitoring of bone marrow purging. Associated new technologies include genetic prodrug activation therapy and differential display polymerase chain reaction, the latter allowing catalogues of gene expression to be prepared for individual tumours. Gene therapy has yet to find proven applications in daily use, but early clinical trials are now in progress. For example, a study of immunotherapy for cervical cancer is underway, using a recombinant vaccine encoding human papilloma virus genes E6 and E7 to generate specific cytotoxic $\mathrm{T}$ cells in vivo.

\section{Conclusions}

Advances in our knowledge of human tumour biology and the workings of the human genome have given us a much clearer understanding of the complexities of solid tumours, but have yet to translate into effective therapies. New technologies and progress in molecular biology and the Human Genome Project will continue to advance our understanding. However, hope that this will translate into dramatic progress in therapeutics must be tempered by the recognition of the subtlety of many cancerous changes and the similarities rather than the differences between tumour and normal cells. These narrow the scope for achieving an effective therapeutic ratio using existing and novel therapies, and for minimising the side-effects of existing treatments. Nevertheless, we live in a remarkable era of scientific progress, in which overpessimistic and overoptimistic predictions are equally unwise!

It has been necessary to be selective, often in an arbitrary fashion, in the inclusion of references for this article. Acknowledgement is due to the many authors whose work is addressed either directly or indirectly but not quoted here.

mediates the suppression of metastases by a Lewis lung carcinoma. Cell 1994;79:315-28.

1 Rew DA Heterogeneity, biodiversity and bioperversity in human solid tumours. Eur $\mathcal{F}$ Surg Oncol 1996;22:469-73.

2 Vogelstein B, Fearon ER, Hamilton SR, et al. Genetic alterations during colorectal tumour development. N Engl ₹ Med 1988;319:525-32.

3 Rennie J. Immortal's enzyme. Sci Am 1994;271: $8-9$.

4 Hartwell LH, Kastan MB. Cell cycle control and cancer. Science 1994;266:1821-8.

5 Peters G. Cell cycle: stifled by inhibitions. Nature 1994;371:204-5.

6 Rew DA. Significance of aneuploidy. Br f Surg 1994;81:1416-22.

7 Kerr JFR, Wyllie AH, Currie AH. Apoptosis, a basic biological phenomenon with wider implications in tissue kinetics. $\mathrm{Br} \mathcal{F}$ Cancer 1972;26: 239-45.

8 Nagata S. Apoptosis by death factor. Cell 1997 ; 88:355-65.

9 Harris CC. p53: at the crossroads of molecular carcinogenesis and risk assessment. Science 1993;262:1980-1.

10 Levine AJ. p53, the cellular gatekeeper for growth and division. Cell 1997;88:323-31.

11 Minden MD, Pawson AJ. Oncogenes. In: Tannock I, Hill RP, eds. The basic science of Tannock I, Hill RP, eds. The basic science of
oncology, 2nd edn. New York: McGraw Hill, oncology, 2nd ed
1992; pp 61-87.

12 Hunter A. Oncoprotein networks. Cell 1997;88: 333-46.

13 Garrod DR. Cell to cell and cell to matrix adhesion. BMF 1993;306:703-5.

14 Menger MD, Vollmar B. Review: Adhesion molecules as determinants of disease: from molecular biology to surgical research. Brf Surg 1996;83:588-601.

15 O'Reilly MS, Holmgren L, Shing Y, et al. Angiostatin: a novel angiogenesis inhibitor that
16 Fidler IJ, Ellis LM. The implications of angiogenesis for the biology and therapy of cancer metastasis. Cell 1994;79:185-8.

17 Folkman J. Angiogenesis in cancer, vascular and rheumatoid disease. Nat Med 1995;1:27-31.

18 Jiang WG, Puntis MCA, Hallett MB. Molecular and cellular basis for cancer invasion and metastasis: implications for treatment. Br $₹$ Surg metastasis: implica

19 Skipper HE, Schabel FM, Wilcox WS. Experimental evaluation of potential anti-cancer agents XII. On the criteria and kinetics associated with curability of experimental leukemia. Cancer Chemother Rep 1964;35:1-111.

20 Tannock I. Experimental chemotherapy and concepts related to the cell cycle. Int $\mathcal{f}$ Radia Biol 1986;49:335-55.

21 Simpson-Herren L. Kinetic perturbations during cancer therapy. Ann NY Acad Sci 1982;397: 88-100.

22 Rew DA, Wilson GD, Taylor I, Weaver PC. The in vivo proliferation kinetics of human colorectal cancer. Br f Surg 1991;78:60-6.

23 Potten CS, Kellett M, Roberts S, Rew DA, Wilson GD. Measurement of in vivo proliferation in human colorectal mucosa using bromodeoxyuhuman colorectal mucosa

24 Dive C, Evans CA, Whetton $\mathrm{AD}$. Induction of Dive $\mathrm{C}$, Evans $\mathrm{CA}$, Whetton $\mathrm{AD}$. Induction of
apoptosis-new targets for cancer chemotherapy. Semin Cancer Biol 1992;3:417-27.

25 Fisher DE. Apoptosis in cancer therapy: crossing the threshold. Cell 1994;78:539-42.

26 Gibbs JB, Oliff A. Pharmaceutical research in molecular oncology. Cell 1994;79:193-8.

27 DeVita VT. The relationship between tumour mass and resistance to chemotherapy. Cancer
1983;51:1209-20.
28 Jain RK. Barriers to drug delivery in solid tumours. Sci Am 1994;271:42-9.

$29 \mathrm{Liu}$ LF. DNA topoisomerase poisons as antitumour drugs. Annu Rev Biochem 1989;58:351 75 .

30 Smith PJ. Topoisomerase inhibitors: new twists to anticancer drug action. Oncol Today 1991;3 4-9.

31 Koshland DE. Molecule of the year: the DNA repair enzyme (editorial). Science 1994;266: 1925-7.

32 Hedley DW. Flow cytometric assays of anticancer drug resistance. Ann NY Acad Sci 1993;677 341-53.

33 Gottesman MM, Pastan I. Multidrug resistance -a review. Annu Rev Biochem 1993;62:385427.

34 Hill RP. Cellular basis of radiotherapy. In: Tannock I, Hill RP. The basic science of oncology, 2nd edn. New York: McGraw Hill, 1992, pp 259-75.

35 Denekamp J. Cell kinetics and radiation biology. Int $f$ Radiat Biol 1986;49:357-80.

36 Rankin EM and McVie JG. Radioimmunodetection of cancer: problems and potential. $B M \mathcal{F}$ 1983;287:1402-4

37 Russell SJ, Llewelyn MB, Hawkins RE. Principles of antibody therapy. BMF 1992;305:1424 28.

38 Ledermann JA. Radiolabelled antibodies for cancer therapy. Hosp Update 1989;15:56-65.

39 Hawkins RE, Llewelyn MB and Russell SJ. Adapting antibodies for clinical use. $B M F 1992$ 305:1348-52.

40 Sikora K. Genes, dreams and cancer. $B M \mathcal{F}$ 1994;308:1217-21. 